\title{
Peridynamic modeling of composite laminates under explosive loading
}

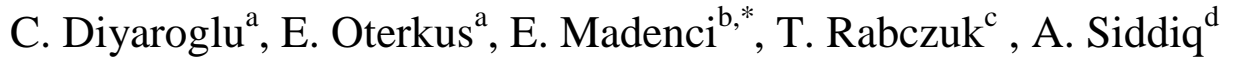 \\ ${ }^{a}$ Department of Naval Architecture and Marine Engineering, University of Strathclyde, Glasgow G4 OLZ, United \\ Kingdom \\ ${ }^{b}$ Department of Aerospace and Mechanical Engineering, The University of Arizona, Tucson, AZ 85721, United \\ States of America \\ ${ }^{c}$ Institute of Structural Mechanics, Bauhaus-University, Weimar, 99423, Germany \\ ${ }^{d}$ School of Engineering, University of Aberdeen, Aberdeen, AB24 3UE, United Kingdom
}

\begin{abstract}
High velocity impact and shock or blast responses are a critical design characteristic determining sizing of composite parts and, ultimately, weight savings. This study demonstrates the applicability of peridynamics to accurately predict nonlinear transient deformation and damage behavior of composites under shock or blast types of loadings due to explosions. The peridynamic predictions correlate well with the experimental results available in the literature. Therefore, peridynamics provides the ability to predict residual strength and durability for improving structural designs of composites under such loading conditions.
\end{abstract}

Keywords: Composites, blast, progressive, failure, peridynamics

\footnotetext{
* Corresponding author. Tel.: +15206216113.

E-mail addresses: cagan.diyaroglu@strath.ac.uk (C. Diyaroglu), erkan.oterkus@ @strath.ac.uk (E. Oterkus), madenci@email.arizona.edu (E. Madenci), timon.rabczuk@uni-weimar.de (T. Rabczuk), amir.siddiq@abdn.ac.uk (A. Siddiq).
} 


\section{Introduction}

During the service life of an aircraft composite component, damage due to high velocity impact or blast may occur, which leads to catastrophic failure of these structures. However, component-level structural testing and analysis of advanced composites is prohibitively expensive and time consuming. Therefore, using robust and accurate computational tools complemented by experiments at key stages is a viable and cost-effective option.

High velocity impact and blast loads cause nonlinear structural deformation and multifaceted failure mechanism in composite laminates. However, it is a very challenging task to predict all possible failure modes because damage initiation and its progressive growth is very complex, and commonly accepted methods have had limited success. It is evident that the inhomogeneous nature of composites must be retained in the analysis to predict the correct failure modes. Aside from the complex loading conditions, the deformation of a laminate is dependent on the lamina properties, thickness, and stacking sequence. There exists, usually, a resin-rich and extremely thin layer between the laminae; an inherent source for cracking and delamination. Therefore, transverse normal and shear deformations especially play a critical role in the initiation and growth of delamination.

High velocity impact and shock or blast responses are a critical design characteristic determining the sizing of composite parts and, ultimately, weight savings. Deformation and failure characteristics of composite materials under shock loading conditions were considered in the past as part of many computational/analytical and experimental investigations. Rabczuk et al. [1] developed a simple model with two lumped masses to analyze sandwich structures subjected to dynamic underwater loads. Motley et al. [2] numerically investigated initial failure loads of fully submerged composite plates subjected to explosion by employing Hashin's criteria for 
failure initiation. A more complex study was performed by Batra and Hassan [3] for a composite laminate subjected to underwater shock loading by using a finite element method (FEM) while incorporating a rate-dependent damage evolution equations. Also, LeBlanc [4] used LS-DYNA, a commercially available FE software, which permits specific material models while incorporating progressive damage property. Wei et al. [5] proposed a progressive degradation model in order to analyze different damage mechanisms in composite structures, and they compared their results with experimental observations obtained from an underwater shock tube. Later, these results were improved by considering strain-rate effects on the mechanical behavior of constituents of composites [6]. Experimental investigations were also carried out in order to gain a better understanding of the dynamic and damage behavior of composite structures under shock loadings. In general, experiments were performed under either direct explosions or with laboratory-scale shock tubes. Using shock tubes is more favorable than using explosives [7] because field experiments can be expensive, dangerous, and harmful to the environment [8]. In experiments, small target dimensions may lead to small impacted regions and, subsequently, localized damage [9].

Therefore, scaling relations involving plate dimensions, explosive intensity, and other parameters are rather important. Bachynski et al. [8] derived scaling relations for composite structures to conduct laboratory-scale experiments. Espinosa et al. [10] developed a novel experimental setup, which is based on scaling analysis, in order to represent full field experiments on a laboratory scale. Other shock tube test setups have been used in the literature $[4,11-13]$ to understand deformation and failure characteristics of composite structures. Mouritz [13] carried out prototype-scale experiments and showed the effect of stitching on improving damage characteristics, especially delamination damage, of glass/vinyl ester composites. Arora 
et al. [7] carried out large-scale field experiments and investigated failure mechanisms of E-glass fiber-reinforced sandwich panels and laminated tubes. Latourte et al. [9] investigated failure modes and damage mechanisms of composite laminate and sandwich structures using a shock tube defined by Espinosa et al. [10]. Avachat and Zhou [14] used a novel gas-gun based Underwater Shock Loading Simulator (USLS) for investigating damage characteristics of composite structures, and comparisons were done with FE simulations performed by Avachat [12].

In summary, several numerical investigations have been performed in collaboration with experimental studies in order to develop the most suitable and accurate numerical modeling technique. However, the previous numerical studies utilized FE analysis, which suffers from mesh sensitivity in the case of impact analyses [15]. Although the use of Cohesive Zone Elements (CZE) is suitable for pure mode I or II type failures, it is still a topic of research for mixed-mode type failure. It requires a priori knowledge of the crack propagation path for CZE placement. In the case of composites, it is also not practical to place CZEs in between each ply for delamination and in-plane matrix cracking. Moreover, they require remeshing for accurate predictions, which is computationally challenging. While the eXtended Finite Element Method (XFEM) has been successfully applied to numerous applications with a moderate number of cracks, its application to complex fracture patterns as they occur in blast events of composite structures remains a challenge. Furthermore, although XFEM is capable of modeling crack growth without remeshing, it still requires a criterion for crack branching and coalescence, and robust criteria for such cases are still missing.

Meshfree methods [16] have been shown to be a good alternative to the finite element method for problems involving large deformations, fracture, and fragmentation. They can handle 
changes in the 'nodal connectivities' more naturally. For example, modeling perforation during impact requires the deletion of elements. Meshfree methods have been extensively applied to dynamic fracture and fragmentation since the nineties [17-19]. They have been used to model shear bands in metals [20-23], concrete fragmentation [24-26], dynamic fracture in thin shells [21, 27-28], and fluid structure interaction [29, 30], among others.

Early approaches were based on Eulerian kernels, where fracture is modeled through a natural separation of particles. However, it was shown for instance in [31] that the use of Eulerian kernels lead to numerical fracture — that could be avoided by formulations based on Lagrangian kernels [32] — and would, in turn, require fracture criteria and a representation of the crack topology. A simple and robust method to treat dynamic fracture that does not require a representation of the crack topology was presented by Sulsky et al. [33]. The Cracking Particles Method (CPM) $[24,34]$ was specifically designed for complex fracture patterns such as crack branching and coalescence. In the CPM, the crack path is represented by a set of cracked particles. The crack kinematics, which is assumed to be piecewise constant, is obtained through enrichment, though a simple particle splitting [35, 36] can achieve the same objective.

Silling $[37,38]$ introduced a nonlocal theory that does not require spatial derivatives, the peridynamic (PD) theory. This theory provides nonlinear material response with respect to displacements. Furthermore, the material response includes damage in the PD theory. The PD theory is formulated by using integral equations, and this feature allows damage initiation and propagation at multiple sites, with arbitrary paths inside the material, without resorting to special crack growth criteria. In the PD theory, internal forces are expressed through nonlocal interactions between the material points within a continuous body, and damage is part of the 
constitutive model. Interfaces between dissimilar materials have their own properties and damage can propagate when and where it is energetically favorable for it to do so.

The PD methodology overcomes the weaknesses of the existing methods, and it is capable of identifying all of the failure modes without simplifying assumptions. It effectively predicts complex failure modes in composites under general dynamic and static loading conditions [3945]. Damage is inherently calculated in a PD analysis without special procedures, making progressive failure analysis more practical. The governing equations of peridynamics are in the form of integro-differential equations, which naturally incorporates damage into the structure, and no additional equations are needed for damage evolution. Moreover, its numerical implementation is achieved by a meshless approach, which does not result in unrealistic energy dissipations as in FEM [15].

This study first briefly explains the peridynamic laminate theory (PDLT) for composite structures by Madenci and Oterkus [46]. Subsequently, it presents simulation results to demonstrate the capability of PD theory for shock/blast type analysis of composites by comparison with the previous experimental study by LeBlanc [4].

\section{Peridynamics}

In the peridynamic theory introduced by Silling [37], and later extended by Madenci and Oterkus [46], the material points interact with each other directly through the prescribed response function, which contains all of the constitutive information associated with the material. The response function includes a length scale parameter called internal length (horizon), $\delta$. The locality of interactions depends on the horizon, and interactions become more local with a decreasing horizon. Hence, the classical theory of elasticity can be considered as a limiting case 
of the peridynamic theory as the internal length approaches zero. The PD theory is a good alternative to traditional methods for damage prediction in materials and structures. As the interactions between material points cease, cracks may initiate and align themselves along surfaces that form cracks, yet the integral equations continue to remain valid.

Madenci and Oterkus [46] extended the PD theory to PD laminate theory, and the details of the derivation and many validation cases are given by Colavito [47]. Each fiber-reinforced composite lamina of a laminate shown in Fig. 1 is idealized as a two-dimensional structure with the directional dependency of the interactions between the peridynamic material points.

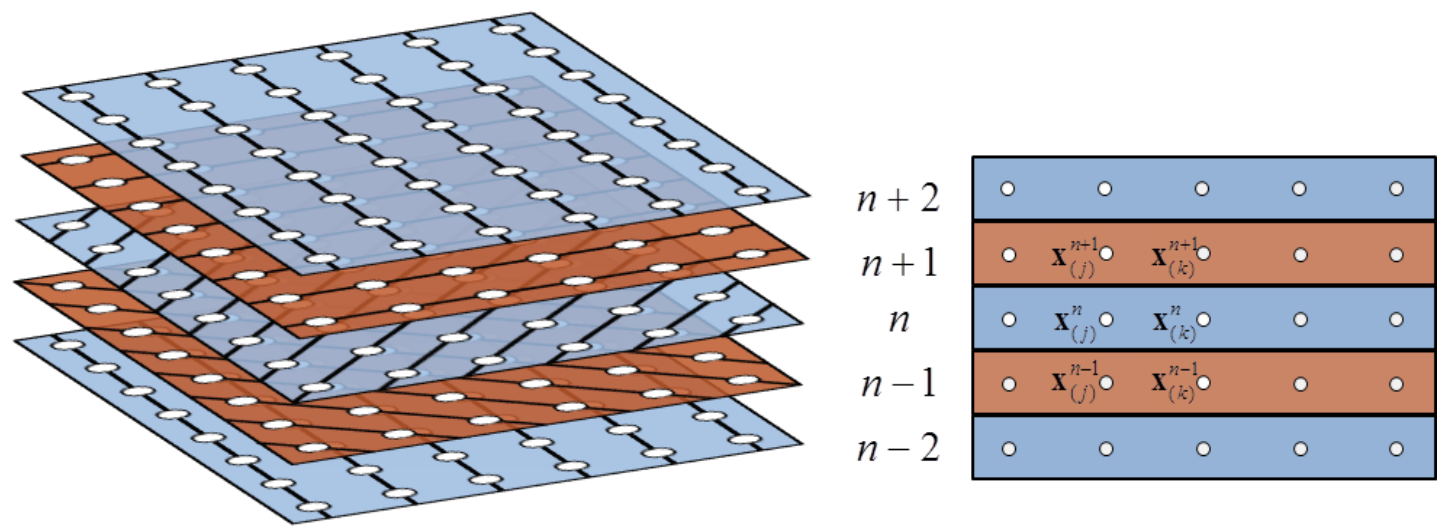

Fig. 1.Elevation of each lamina in a laminate and PD material points.

As shown in Fig. 2, the material point $q$ represents material points that interact with material point $k$ only along the fiber direction with an orientation angle of $\theta$ in reference to the $x$-axis. Similarly, material point $r$ represents material points that interact with material point $k$ only along the transverse direction. However, the material point $p$ represents material points that interact with material point $k$ in any direction, including the fiber and transverse directions. The orientation of a PD interaction between the material point $k$ and the material point $p$ is defined by the angle $\phi$ with respect to the $x$-axis. The domain of integration, $H$ shown in Fig. 2, is a 
disk with radius $\delta$ and thickness $h$. The material points in a particular lamina interact with the other material points of immediate neighboring laminae, above and below it.

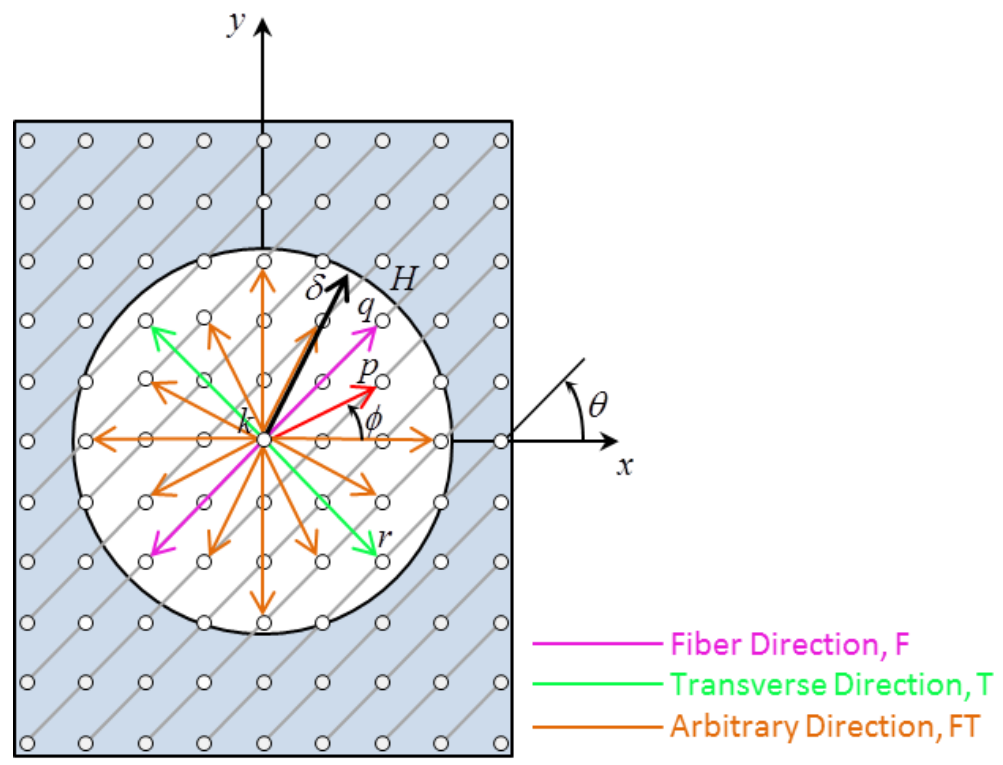

Fig. 2.PD horizon for a family of material points and their interactions in a lamina.

The equation of motion for material point $\mathbf{x}_{(k)}^{n}$ located on the $n^{\text {th }}$ layer of a laminate with $N$ layers can be expressed as

$$
\begin{aligned}
& \rho_{(k)}^{n} \ddot{\mathbf{u}}_{(k)}^{n}=\sum_{j=1}\left[\mathbf{t}_{(k)(j)}^{n}\left(\mathbf{u}_{(j)}^{n}-\mathbf{u}_{(k)}^{n}, \mathbf{x}_{(j)}^{n}-\mathbf{x}_{(k)}^{n}, t\right)-\mathbf{t}_{(j)(k)}^{n}\left(\mathbf{u}_{(k)}^{n}-\mathbf{u}_{(j)}^{n}, \mathbf{x}_{(k)}^{n}-\mathbf{x}_{(j)}^{n}, t\right)\right] V_{(j)}^{n} \\
& +\sum_{m=n+1, n-1} \mathbf{p}_{(k)}^{(n)(m)} V_{(k)}^{m}+\sum_{m=n+1, n-1} \sum_{j=1} 2 \mathbf{q}_{(k)(j)}^{(n)(m)} V_{(j)}^{m}+\mathbf{b}_{(k)}^{n}
\end{aligned}
$$

where the material point $\mathbf{x}_{(k)}^{n}$ on the $n^{\text {th }}$ layer is associated with an incremental volume, $V_{(k)}^{n}$, and a mass density of $\rho_{(k)}^{n} ; t$ designates time. With respect to a Cartesian coordinate system, the material point $\mathbf{x}_{(k)}^{n}$ experiences displacement, $\mathbf{u}_{(k)}^{n}$, and its location is described by the position 
vector $\mathbf{y}_{(k)}$ in the deformed state. The displacement and body load vectors at material point $\mathbf{x}_{(k)}^{n}$, are represented by $\mathbf{u}_{(k)}^{n}$ and $\mathbf{b}_{(k)}^{n}$, respectively.

Arising from in-plane deformation, $\mathbf{t}_{(k)(j)}^{n}$ represents the force density that material point $\mathbf{x}_{(j)}^{n}$ exerts upon material point $\mathbf{x}_{(k)}^{n}$. The force density vectors, $\mathbf{p}_{(k)}^{(n)(m)}$ and $\mathbf{q}_{(k)(j)}^{(n)(m)}$ with $m=(n+1),(n-1)$, develop due to the transverse normal and transverse shear deformations, respectively, between the material points $\mathbf{x}_{(k)}^{n}$ and $\mathbf{x}_{(j)}^{m}$. The explicit form of the force density vectors, $\mathbf{t}_{(k)(j)}^{n}, \mathbf{p}_{(k)}^{(n)(m)}$ and $\mathbf{q}_{(k)(j)}^{(n)(m)}$ associated with in-plane, transverse normal, and shear deformations, respectively, can be derived in the form

$\mathbf{t}_{(k)(j)}^{(n)}=2 \delta\left(a d \frac{\Lambda_{(k)(j)}^{n}}{\left|\mathbf{x}_{(j)}^{n}-\mathbf{x}_{(k)}^{n}\right|} \theta_{(k)}^{n}+\left(\mu_{F} b_{F}+b_{F T}+\mu_{T} b_{T}\right) s_{(k)(j)}^{(n)}\right) \frac{\mathbf{y}_{(j)}^{n}-\mathbf{y}_{(k)}^{n}}{\left|\mathbf{y}_{(j)}^{n}-\mathbf{y}_{(k)}^{n}\right|}$

$\mathbf{p}_{(k)}^{(n)(m)}=4 b_{N} \hat{\delta}\left(\frac{\left|\mathbf{y}_{(k)}^{m}-\mathbf{y}_{(k)}^{n}\right|-\left|\mathbf{x}_{(k)}^{m}-\mathbf{x}_{(k)}^{n}\right|}{\left|\mathbf{x}_{(k)}^{m}-\mathbf{x}_{(k)}^{n}\right|}\right) \frac{\mathbf{y}_{(k)}^{m}-\mathbf{y}_{(k)}^{n}}{\left|\mathbf{y}_{(k)}^{m}-\mathbf{y}_{(k)}^{n}\right|}$

$\mathbf{q}_{(k)(j)}^{(n)(m)}=4 b_{S} \tilde{\delta}\left[\left(\frac{\left|\mathbf{y}_{(j)}^{m}-\mathbf{y}_{(k)}^{n}\right|-\left|\mathbf{x}_{(j)}^{m}-\mathbf{x}_{(k)}^{n}\right|}{\left|\mathbf{x}_{(j)}^{m}-\mathbf{x}_{(k)}^{n}\right|}\right)-\left(\frac{\left|\mathbf{y}_{(k)}^{m}-\mathbf{y}_{(j)}^{n}\right|-\left|\mathbf{x}_{(k)}^{m}-\mathbf{x}_{(j)}^{n}\right|}{\left|\mathbf{x}_{(k)}^{m}-\mathbf{x}_{(j)}^{n}\right|}\right)\right] \frac{\mathbf{y}_{(j)}^{m}-\mathbf{y}_{(k)}^{n}}{\left|\mathbf{y}_{(j)}^{m}-\mathbf{y}_{(k)}^{n}\right|}$

with

$s_{(k)(j)}^{(n)}=\frac{\left|\mathbf{y}_{(j)}^{n}-\mathbf{y}_{(k)}^{n}\right|-\left|\mathbf{x}_{(j)}^{n}-\mathbf{x}_{(k)}^{n}\right|}{\left|\mathbf{x}_{(j)}^{n}-\mathbf{x}_{(k)}^{n}\right|}$ 
and

$$
\Lambda_{(k)(j)}^{n}=\frac{\mathbf{y}_{(j)}-\mathbf{y}_{(k)}}{\left|\mathbf{y}_{(j)}-\mathbf{y}_{(k)}\right|} \cdot \frac{\mathbf{x}_{(j)}-\mathbf{x}_{(k)}}{\left|\mathbf{x}_{(j)}-\mathbf{x}_{(k)}\right|}
$$

which represents the direction cosines of the relative position vectors between the material points $\mathbf{x}_{(k)}^{n}$ and $\mathbf{x}_{(j)}^{n}$ in the undeformed and deformed states.

The horizon size in the thickness direction is $\hat{\delta}$, and $\tilde{\delta}$ is defined as $\tilde{\delta}=\sqrt{\delta^{2}+\hat{\delta}^{2}}$. Note that $\left|\mathbf{x}_{(j)}^{m}-\mathbf{x}_{(k)}^{n}\right|$ and $\left|\mathbf{x}_{(k)}^{m}-\mathbf{x}_{(j)}^{n}\right|$ are equivalent quantities. The parameters $\mu_{F}$ and $\mu_{T}$ define the direction of the fiber as

$$
\mu_{F}=\left\{\begin{array}{ll}
1 & \left(\mathbf{x}_{(j)}-\mathbf{x}_{(k)}\right) / / \text { fiber direction } \\
0 & \text { otherwise }
\end{array} \quad \mu_{T}= \begin{cases}1 & \left(\mathbf{x}_{(j)}-\mathbf{x}_{(k)}\right) \perp \text { fiber direction } \\
0 & \text { otherwise }\end{cases}\right.
$$

The parameter $\theta_{(k)}$ denotes the dilatation of the material point $\mathbf{x}_{(k)}^{n}$ and is defined as

$$
\theta_{(k)}^{n}=d \sum_{j=1}^{\infty} \frac{\delta}{\left|\mathbf{x}_{(j)}^{n}-\mathbf{x}_{(k)}^{n}\right|}\left(\left|\mathbf{y}_{(j)}^{n}-\mathbf{y}_{(k)}^{n}\right|-\left|\mathbf{x}_{(j)}^{n}-\mathbf{x}_{(k)}^{n}\right|\right) \Lambda_{(k)(j)}^{n} V_{(j)}^{n}
$$

The PD material parameters $a$ and $d$ are associated with the dilatation term, and $b_{F}, b_{T}$, and $b_{F T}$ are associated with deformation of PD interactions in the fiber direction, transverse direction, and remaining arbitrary directions, respectively. The PD material parameters $b_{N}$ and 
$b_{s}$ are associated with the transverse normal and shear deformations. These parameters can be related to the four independent material constants of elastic modulus in the fiber direction, $E_{11}$, elastic modulus in the transverse direction, $E_{22}$, in-plane shear modulus, $G_{12}$, and in-plane Poisson's ratio, $v_{12}$. Their explicit expressions are derived by Madenci and Oterkus [ 46].

This peridynamic laminate theory described in detail by Madenci and Oterkus [46] includes transverse normal and shear deformations, and it accurately models the behavior of fiberreinforced composites. It accounts for deformation coupling such as stretch-shear, stretchtwisting, and stretch-bending due to material layup. It is also geometrically nonlinear and captures damage through the thickness in the presence of local loading, such as blast or shock due to explosion.

Since the force density-stretch relations are nonlinear, the peridynamic equation of motion is solved numerically. Therefore, in order to carry out the numerical integration, the region of interest is first discretized into sub-domains in which the displacement and velocity fields are assumed to be constant. Hence, each sub-domain can be represented as a single collocation point located at the mass center of the sub-domain.

\subsection{Peridynamics for progressive damage}

The material point $\mathbf{x}$ has in-plane interactions within the same ply, as well as interlayer interactions between the adjacent plies above and below, as shown in Fig. 2. The interactions within the same layer establish the in-plane properties of the composite laminate, including fiber and matrix. The interlayer interactions with material points from different layers define the interlayer properties. The delamination prediction is based on the deformation state of the interlayer; they are terminated once reaching their critical values. Local damage at a point is 
defined as the weighted ratio of the number of eliminated interactions to the total number of initial interactions of a material point with its family members. The local damage at a point can be quantified as [48]

$\varphi(\mathbf{x}, t)=1-\frac{\int_{H} \mu\left(\mathbf{x}^{\prime}-\mathbf{x}, t\right) d V^{\prime}}{\int_{H} d V^{\prime}}$

The status variable, $\mu$, is defined as

$\mu=\left\{\begin{array}{lll}1, & s<s_{c} & \text { no damage } \\ 0, & s \geq s & \text { damage }\end{array}\right.$

with $s_{c}$ representing the critical value of stretch. The local damage ranges from zero to one. When the local damage is one, all the interactions initially associated with the point have been eliminated, while a local damage of zero means that all interactions are intact. The measure of local damage is an indicator of possible crack formation within a body.

\subsubsection{Intralayer damage}

The constitutive or force-stretch relations for the interactions within the plane of a lamina, in the fiber, transverse, and arbitrary directions, are shown in Fig. 3. In this study, the transverse and arbitrary critical parameters are combined into a matrix critical stretch in tension and compression as $s_{m t}$ and $s_{m c}$, respectively. The critical parameters in the fiber direction in tension 
and compression are $s_{f t}$ and $s_{f c}$, respectively. The critical stretch parameters for composite laminates can be obtained using experimental methods, Oterkus et al. [39, 40], calibration using an inverse approach $[49,50]$, or through equating the energy required to create a fracture surface to the energy release rate [51].

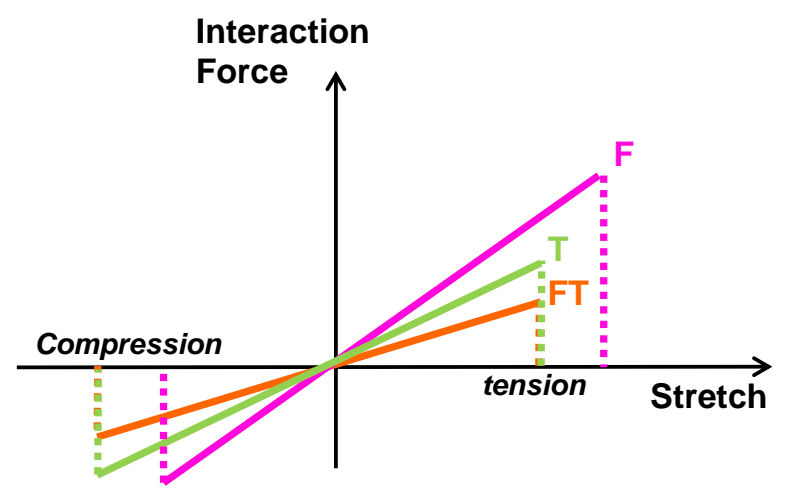

Fig. 3. Force-stretch relationships for peridynamic interactions.

\subsubsection{Interlayer damage}

The interlayer interactions may break due to the opening mode, releasing strain energy, $G_{I}$, that accounts for interlaminar tension. Also, interlayer interactions may break due to the shearing mode, and releasing energy, $G_{I I}$, that accounts for interlaminar sliding shear. As suggested by Oterkus and Madenci [39], the transverse normal and the transverse shear critical stretch values can be determined as

$$
s_{d n}=\sqrt{\frac{2 G_{I C}}{h E_{m}}}
$$

and 


$$
s_{d s}=\sqrt{\frac{G_{I I C}}{h G_{m}}}
$$

where $G_{I C}$ and $G_{I I C}$ are the mode I and mode II critical energy release rates of the matrix material. The underlying idea is that energies required to break all interlayer interactions between the plies of a laminate are the same with mode I or mode II critical energy release rates. The elastic and shear moduli of the epoxy matrix material are denoted by $E_{m}$ and $G_{m}$.

\subsection{Numerical time integration}

The numerical solution of the equations of motion is achieved by employing explicit time integration schemes. The advantage of explicit schemes over implicit time integration methods is that there is no need to solve the equation of motion using large matrices because each equation related to the main material point, $i$, can be solved independently. However, the explicit schemes are stable only if the time step size, $\Delta t$, is smaller than a particular value. The stability condition

for isotropic materials was given by Silling and Askari [48]. Similarly, the stability criterion on the time step size can be derived by using a von Neumann stability analysis for a composite laminate as

$$
\Delta t<\sqrt{\frac{2 \rho_{(k)}}{A+B+C}}
$$

in which 


$$
\begin{aligned}
A & =\sum_{j}\left\{2 a d \delta\left[d \delta \sum_{\ell}\left(\frac{1}{\left|\xi_{(\ell)(k)}\right|}+\frac{1}{\left|\xi_{(\ell)(j)}\right|}\right) V_{(\ell)}\right]+4 \delta\left(\mu_{F} b_{F}+b_{F T}+\mu_{T} b_{T}\right)\right\} \frac{1}{\left|\xi_{(k)(j)}\right|} V_{(j)} \\
B & =\sum_{m} 4 b_{N} \hat{\delta} \frac{1}{\left|\mathbf{x}_{(k)}^{m}-\mathbf{x}_{(k)}^{n}\right|} V_{(m)} \\
C & =\sum_{m} 4 b_{S} \tilde{\delta} \frac{1}{\left|\mathbf{x}_{(j)}^{m}-\mathbf{x}_{(k)}^{n}\right|} V_{(m)}
\end{aligned}
$$

The use of a safety factor that has a value of less than 1 is recommended as it makes the analysis more stable in case of some type of nonlinearity in the structure.

\section{Numerical results}

The applicability of this approach is demonstrated by simulating the damage evolution in a 13-ply composite laminate under shock-type loading. The PD predictions are compared against an experimental study performed by LeBlanc [4]. A Conical Shock Tube (CST) was used to replicate underwater shock phenomena. The geometry and mechanical properties of the composite plate are the same as those reported by LeBlanc. The CST experimental setup is shown in Fig. 4. Shock wave propagates from the breech, at which the charge is located, and strikes the test plate. The test plate, shown in Fig 5, is clamped along the boundary region using bolts.

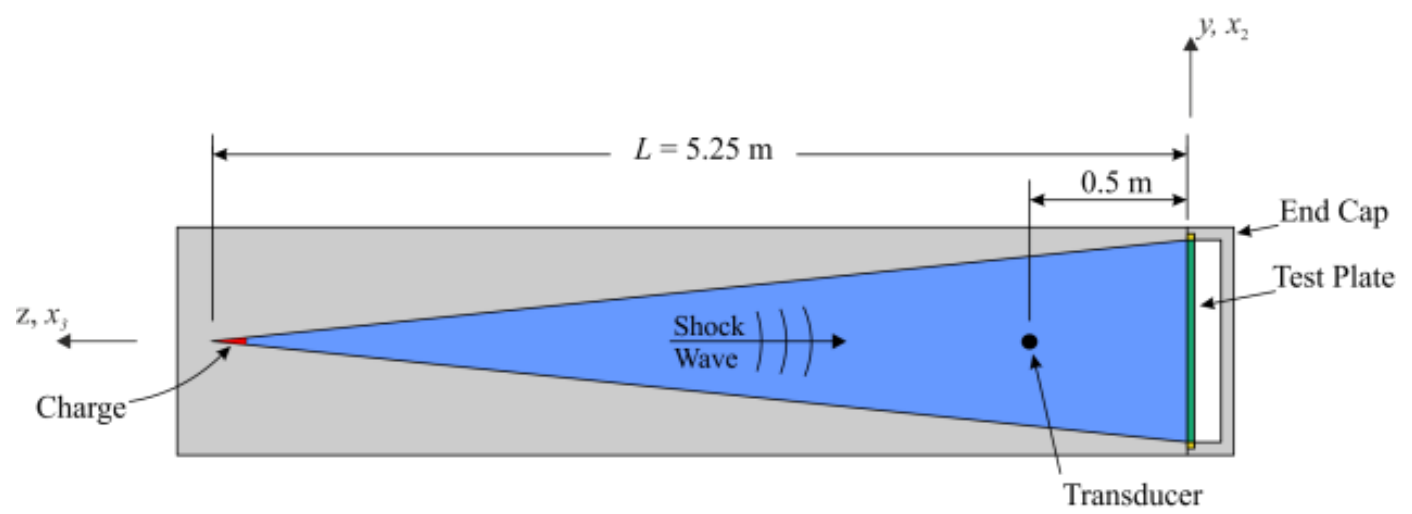

Fig. 4. Representative CST test setup [3]. 


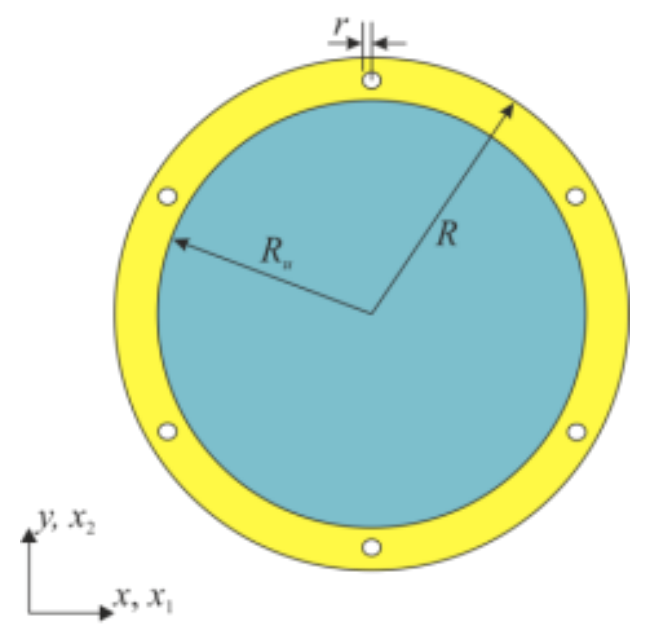

Fig. 5. Composite test plate.

It is worth mentioning that only the first impact of the shock wave is considered, so pressure generated by the incident shock wave alone is applied to the test plate. Based on the measurements by LeBlanc [4], when the shock wave reaches the test plate, pressure rises up linearly to a peak value, $P_{\max }$, during the time range of $0 \mathrm{~ms} \leq t \leq 0.04 \mathrm{~ms}$. It retains its peak value until $t=0.08 \mathrm{~ms}$, before diminishing exponentially. This pressure profile is shown in Fig. 6.

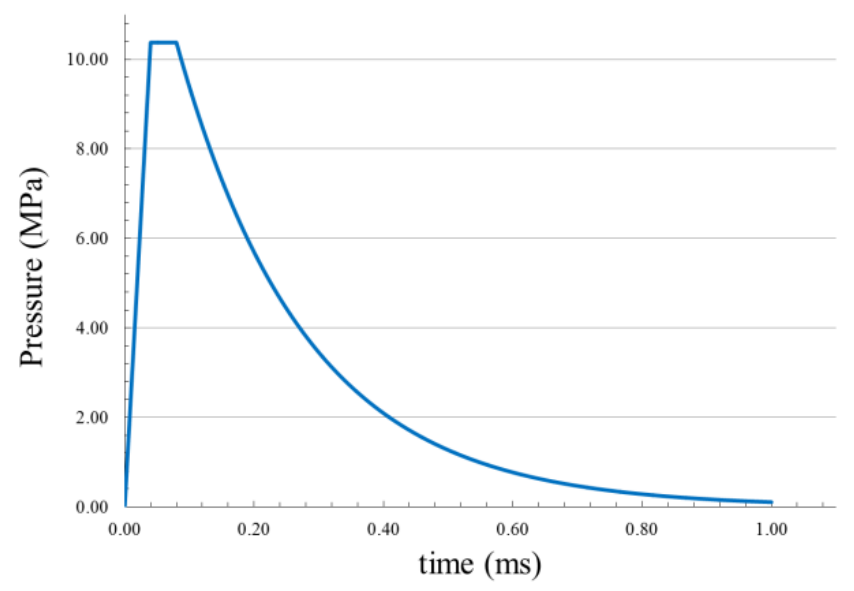

Fig. 6. Pressure profile at the test plate in CST. 
The test plate made of Cyply® 1002 is a 13-ply composite plate with layup of $[0 / 90 / 0 / 90 / 0 / 90 / \overline{0}]$. Each ply is composed of epoxy matrix and E-glass filament materials. The material properties of the unidirectional lamina are $E_{11}=39.3 \mathrm{GPa}, E_{22}=9.7 \mathrm{GPa}$, $G_{12}=9.7 \mathrm{GPa}$, and $v_{12}=0.27$. Its mass density is $\rho=9.7 \mathrm{~kg} / \mathrm{m}^{3}$. Tension and compression strength properties of the lamina are $\sigma_{1 t}=965 \mathrm{MPa}, \sigma_{1 c}=-883 \mathrm{MPa}, \sigma_{2 t}=20 \mathrm{MPa}$, $\sigma_{2 c}=-193 \mathrm{MPa}$. The critical stretch values for fiber failure can be related to the measured tension and compression strength values as $s_{f t}=\sigma_{1 t} / E_{11}$ and $s_{f c}=\sigma_{1 c} / E_{11}$. The critical stretch value, $s_{m t}$, for the matrix under tension is determined as [18]

$$
s_{2 t}=\sqrt{\frac{5 G_{I C}}{9 K_{m} \delta}}
$$

where $G_{I C}$ and $K_{m}$ denote the mode I critical energy release rate and bulk modulus of the epoxy matrix material. The critical energy release rate for the matrix is specified as $G_{I C}=11.85 \times 10^{-3} \mathrm{MPa}-\mathrm{m}$, and the critical stretch value for the matrix direction becomes $S_{m t}=2.1 \times 10^{-2}$. The critical stretch value for fiber is much higher than that of the matrix, thus providing material integrity during the analysis. Also, the critical stretch values in the arbitrary direction are the same as that of the matrix critical stretch. Furthermore, the matrix material is only allowed to fail in tension and not under compression. For the epoxy matrix material between the plies of a laminate, the critical energy release rates, $G_{I C}$ and $G_{I I C}$ for Mode I and Mode II, respectively, are specified as $G_{I C}=2.37 \times 10^{-3} \mathrm{MPa}-\mathrm{m}$ and $G_{I I C}=7.11 \times 10^{-3} \mathrm{MPa}-\mathrm{m}$. 
Thus, the critical stretch values of interlayer damage are computed as $s_{d n}=7.015 \times 10^{-2}$ and $s_{d s}=0.1$.

The PD model of the composite laminate, shown in Fig. 7, includes each lamina with a single layer of material points with a grid size of $\Delta x=1.32715 \times 10^{-3} \mathrm{~m}$. Each material point has a horizon radius of $\delta=3.015 \Delta x$. The fully clamped conditions arising from the mounting fixtures are enforced by constraining only the bottom and top plies in the vertical direction and leaving the remaining free of constraints in other directions.

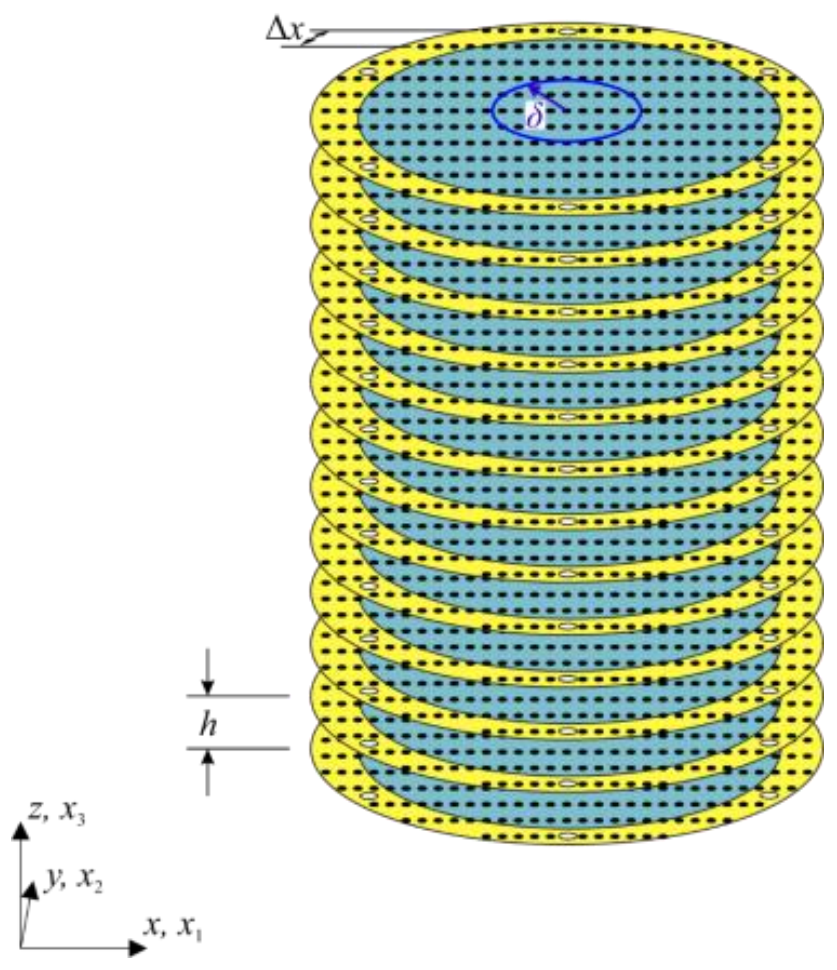

Fig. 7. PD discretization of a 13-ply composite test plate.

Underwater shock analysis of the composite plate is performed for $1 \mathrm{~ms}$; at this instant, the pressure profile becomes nearly zero. However, damage simulation continues until $0.452 \mathrm{~ms}$ because the bolt holes are fragmentized and the plate is pulled apart from the mounting fixtures. The time step size for an explicit time integration is specified as $\Delta t=7.69 \times 10^{-8} \mathrm{~s}$. Peridynamic 
matrix damage and delamination predictions are captured in all 13 plies of the test plate; the plies are numbered from the bottom to top ply as 1 to 13 , respectively.

\subsection{Matrix damage}

Matrix damage predictions are shown in Fig. 8 for all plies at time steps of $0.224 \mathrm{~ms}, 0.325$ $\mathrm{ms}$, and $0.452 \mathrm{~ms}$. Damage progression in the top and bottom plies is very distinctive because the top plies are compressed under shock loading and the bottom plies resist tensile loading. At time $0.224 \mathrm{~ms}$, the loading reaches about half of the maximum applied blast loading. Matrix damage predictions in the top, middle, and bottom plies at each time step are shown in Figs. 9-11. Damage in the bottom plies can be attributed to matrix cracking. However, damage can be both matrix cracking and fiber/matrix debonding near the bolt holes in the top plies. As the time progresses, damage characteristics between the top and bottom plies remain distinct. In comparison to the bottom plies, the matrix damage in the middle region remains lower than that of the top plies. As for the bottom plies, damage increases to higher values at the center of four quadrants. The matrix damage in the vicinity of bolt holes increases as time progresses. It propagates in all plies, and top and bottom bolt holes are fragmentized dramatically. Also, a significant amount of damage propagates towards the center from the top and bottom bolt holes in all plies. Therefore, it leads to the rupture of the plate from the top and bottom clamped regions. 


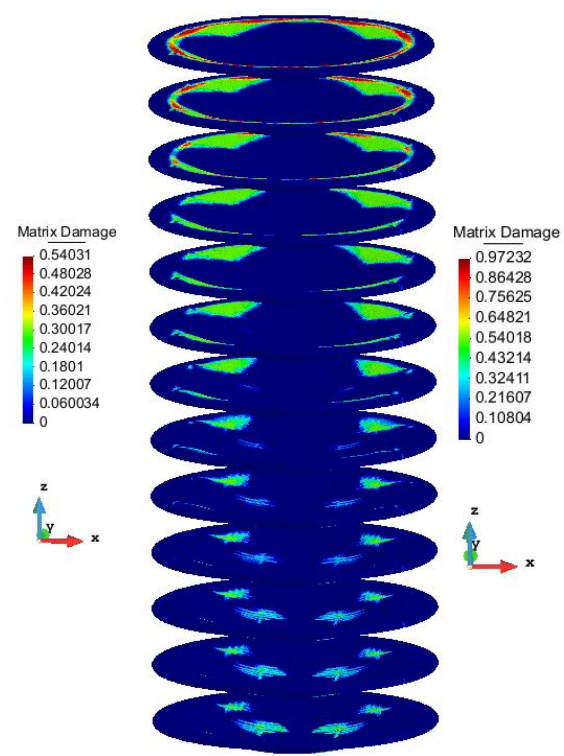

(a)

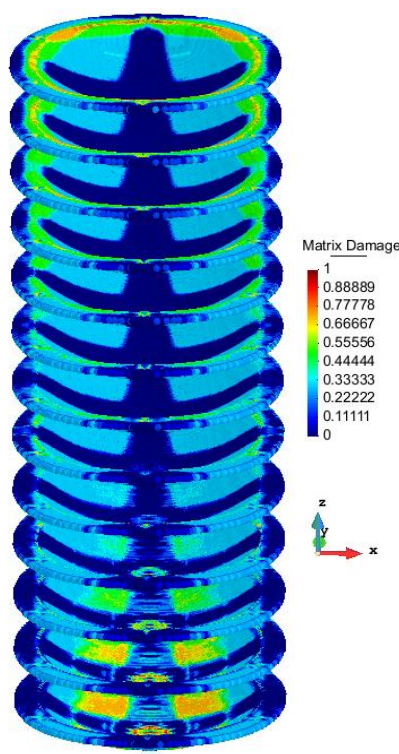

(b)

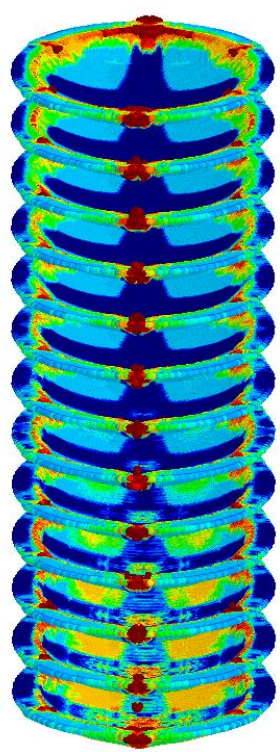

(c)

Fig. 8. Matrix damage results of all plies at (a) $0.224 \mathrm{~ms}$, (b) $0.325 \mathrm{~ms}$, and (c) $0.452 \mathrm{~ms}$.

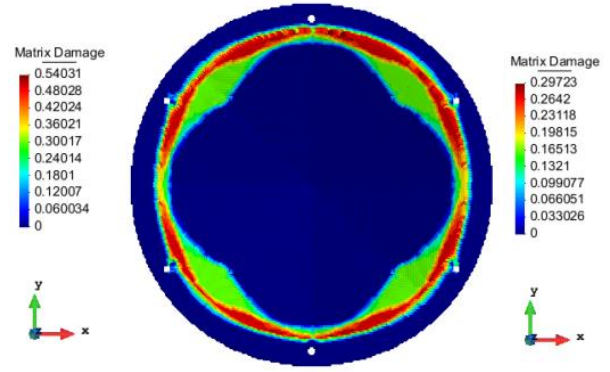

(a)

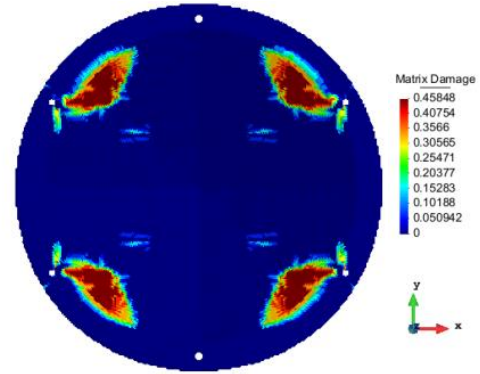

(b)

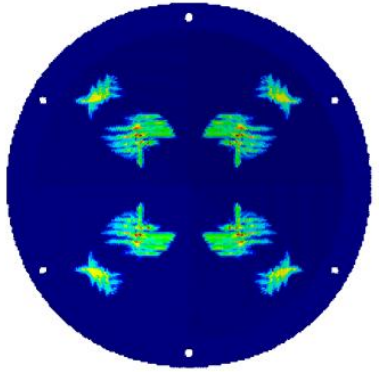

(c)

Fig. 9. Top views of matrix damage of (a) top, (b) middle, and (c) bottom plies at $0.224 \mathrm{~ms}$. 


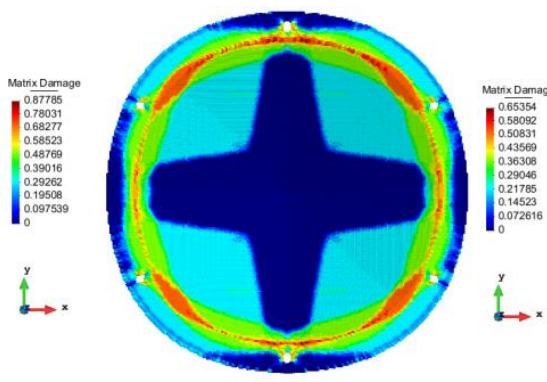

(a)

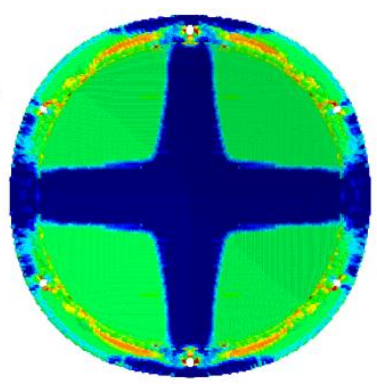

(b)

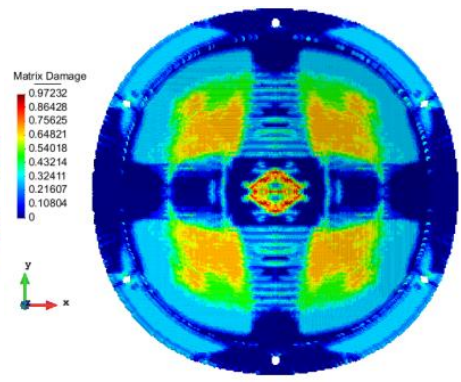

(c)

Fig. 10. Top views of matrix damage of (a) top, (b) middle, and (c) bottom plies at $0.325 \mathrm{~ms}$.

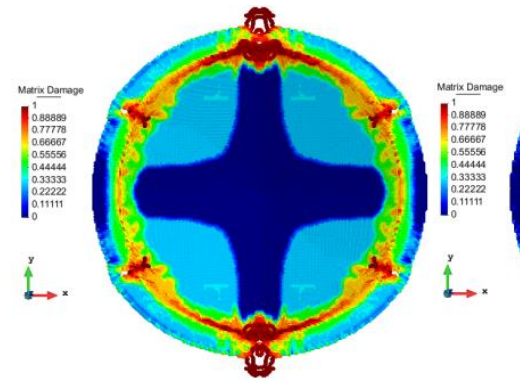

(a)

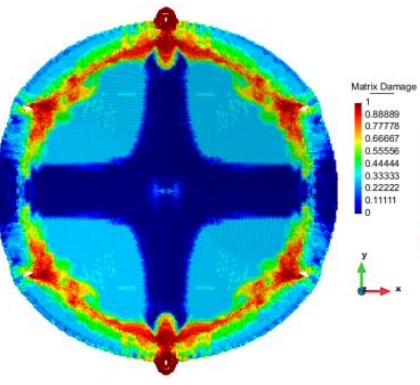

(b)

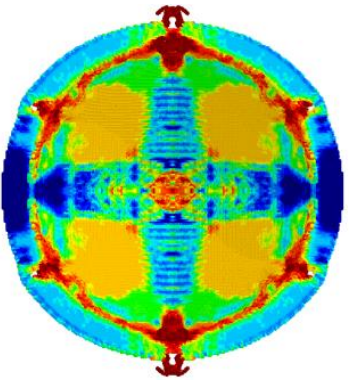

(c)

Fig. 11. Top views of matrix damage of (a) top, (b) middle, (d) bottom plies at $0.452 \mathrm{~ms}$.

\subsection{Delamination damage}

Delamination damage occurs due to either mode I or mode II type deformations. Unlike the matrix damage, both delamination damage types commence at a later time, about $0.325 \mathrm{~ms}$. As shown in Fig. 12a, mode II type delamination damage is observed in the boundary region of all plies; however, it is more considerable in the middle plies. In particular, middle plies have extensive damage around the top, bottom, left, and right sides of the clamped region, as shown in Fig. 13. 


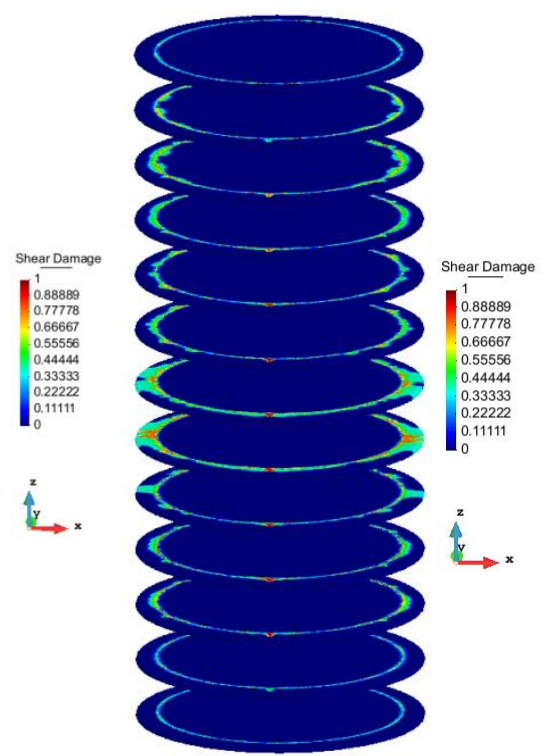

(a)

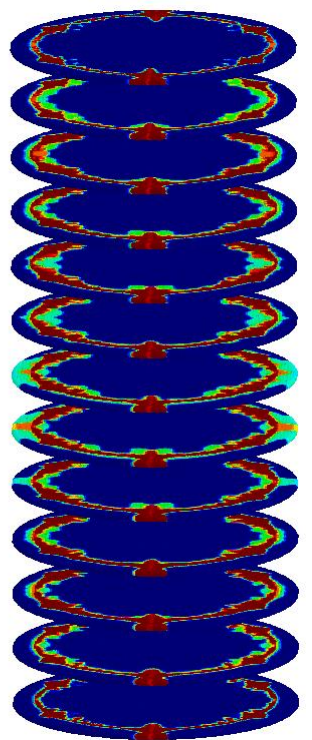

(b)

Fig. 12. Mode II type delamination damage in all plies at $0.325 \mathrm{~ms}$ and $0.452 \mathrm{~ms}$.

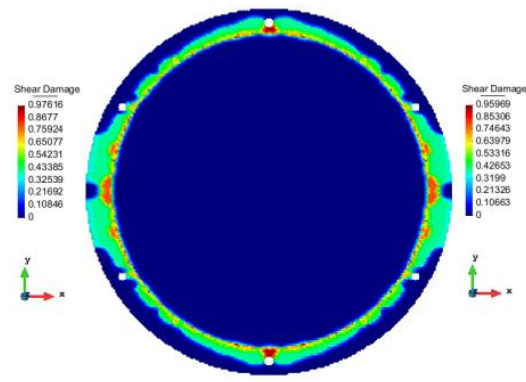

(a)

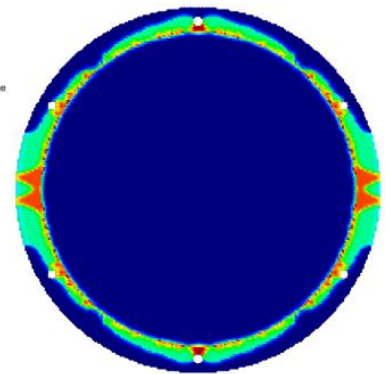

(b)

Fig. 13. Top views of delamination damage for (a) middle and (b) $6^{\text {th }}$ plies at $0.325 \mathrm{~ms}$.

At $0.452 \mathrm{~ms}$, delamination damage propagates, and all plies are delaminated from the circumference of the boundary region, as shown in Fig. 12b, with significant damage concentrations evident around the bolt holes, as shown in Fig. 14. Also, delamination damage propagates towards the center of the plate from the top and bottom bolt holes, similar to that observed in matrix damage predictions at $0.452 \mathrm{~ms}$. Comtois et al. [52] observed similar delamination damage as in Fig. 14 in several experiments of circular specimens due to air shock 
loading. Furthermore, damage propagates towards the inner region from the clamped areas while spreading throughout the specimen with an increase of charge weight.

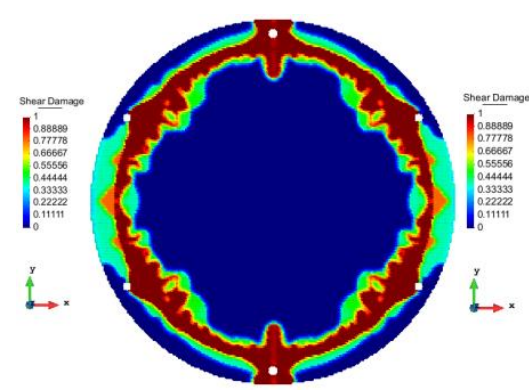

(a)

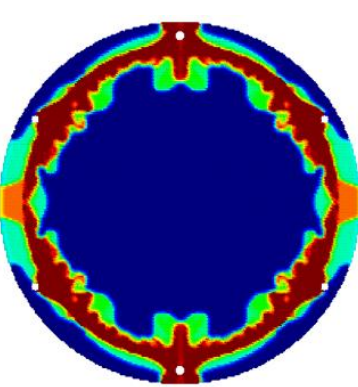

(b)

Fig. 14. Top views of delamination damage for (a) middle and (b) $6^{\text {th }}$ plies at $0.452 \mathrm{~ms}$.

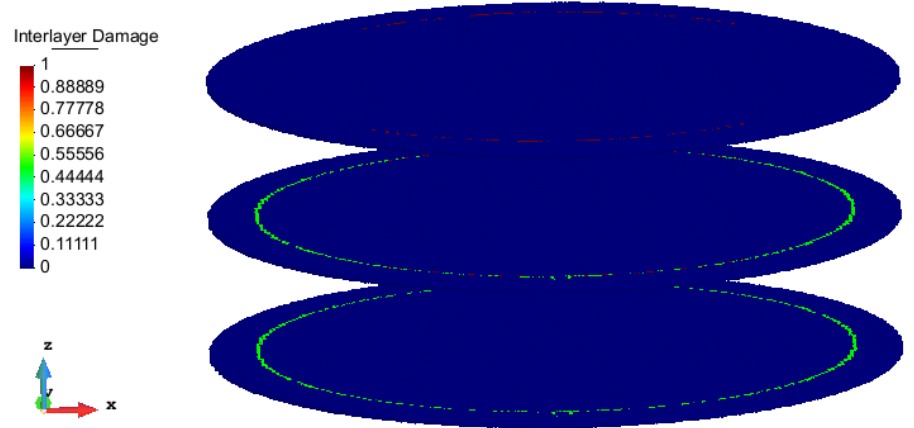

Fig. 15. Mode I type delamination damage in top three plies at $0.325 \mathrm{~ms}$.

Mode I type delamination damage is contained around the circumference of the unsupported region in the top three plies, as shown in Fig. 15. As the time progresses, delamination damage concentrates around the bolt holes and is significant at the top and bottom holes, from which damage spreads along the circumference of the unsupported region especially for the top plies, as can be seen from Figs. 16 and 17. 


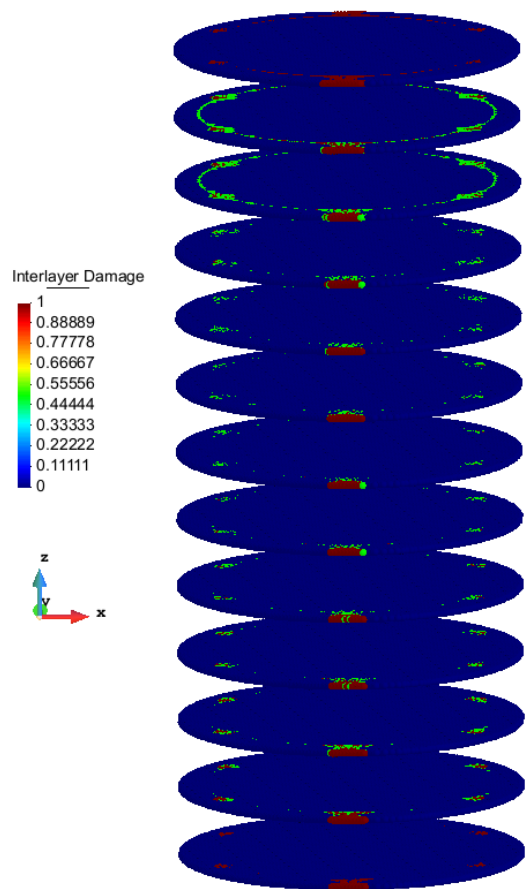

Fig. 16. Mode I type delamination damage in all plies at $0.452 \mathrm{~ms}$.

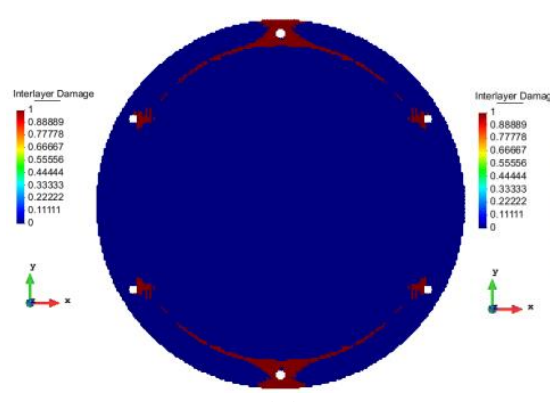

(a)

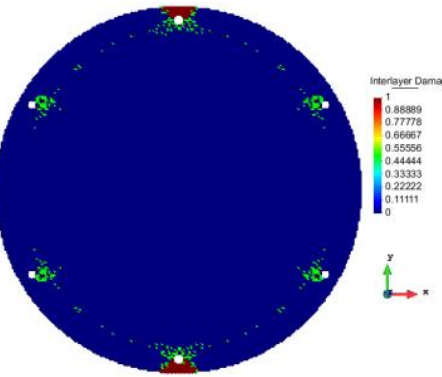

(b)

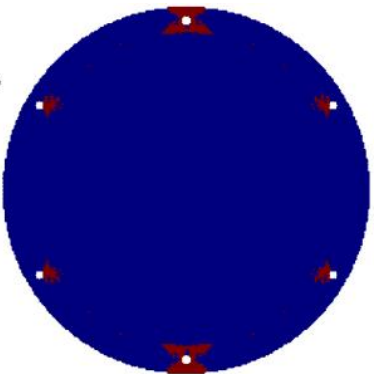

(c)

Fig. 17. Top views of mode I type delamination damage for (a) top, (b) middle, and (c) bottom plies at $0.452 \mathrm{~ms}$.

The PD simulation predicts the test plate to be torn off from the mounting fixtures at 0.452 $\mathrm{ms}$, and it reaches the complete damage state. The predictions are consistent with the image of the damaged test plate provided by LeBlanc [4], shown in Fig. 18. All bolt holes are damaged and the damage around the top and bottom holes is more considerable than the others. Complete 
rupture occurs from the top bolt. Also, damage progressions from the top and bottom holes to the center are quite significant. Similar damage behavior can also be observed in PD matrix damage predictions.

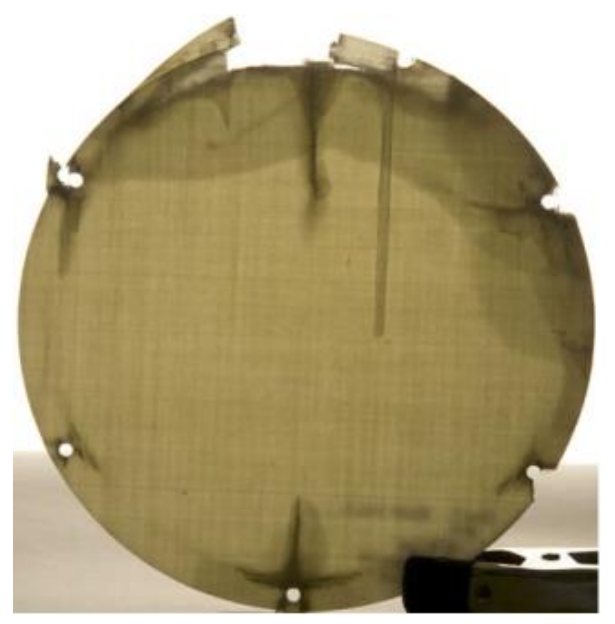

Fig. 18. Damaged test plate after the shock loading in CST [4].

As shown in Fig. 18, transparent regions of the image indicate delamination in the test plate. A considerable amount of delamination can be observed at the top and right sides of the test plate. Also, delamination regions are quite significant at the top and bottom bolt holes. The unsymmetrical delamination damage may be due to the presence of manufacturing process defects. Based on these observations, it can be concluded that the PD simulations successfully capture the experimental observations and delamination regions.

\section{Conclusions}

This study demonstrates the capability of PD theory for the prediction of damage patterns of complex structures under shock loading. The PD methodology overcomes the weaknesses of the existing methods, and it is capable of identifying all of the failure modes without simplifying 
assumptions. Damage is inherently calculated in a PD analysis without special procedures, making progressive failure analysis more practical. The peridynamic predictions correlate well with experimental results available in the literature. Therefore, peridynamics can be used as a very effective computational methodology for investigating high velocity impact and blast response of composite structures.

\section{References}

[1] Rabczuk T, Samaniego E, Belytschko T. A simplified model for predicting impulsive loads on submerged structures to account for fluid-structure interaction. Int J Impact Eng 2007;34:163-177.

[2] Motley MR, Young YL, Liu Z. 2011. Three-Dimensional Underwater Shock Response of Composite Marine Structures. J Appl Mech 2011;78:61013.

[3] Batra RC, Hassan NM. Response of fiber reinforced composites to underwater explosive loads. Compos Part B Eng 2007;38:448-468.

doi:http://dx.doi.org/10.1016/j.compositesb.2006.09.001.

[4] LeBlanc J. Dynamic response and damage evolution of composite materials subjected to underwater explosive loading: an experimental and computational study. Kingston, RI: University of Rhode Island, 2011.

[5] Wei X, de Vaucorbeil A, Tran P, Espinosa HD. A new rate-dependent unidirectional composite model — application to panels subjected to underwater blast. J Mech Phys Solids 2013;61:305-1318. doi:http://dx.doi.org/10.1016/j.jmps.2013.02.006. 
[6] Wei X, Tran P, de Vaucorbeil A, Ramaswamy RB, Latourte F, Espinosa HD. Threedimensional numerical modeling of composite panels subjected to underwater blast. $\mathbf{J}$ Mech Phys Solids 2013;61:1319-1336. doi:http://dx.doi.org/10.1016/j.jmps.2013.02.007.

[7] Arora H, Hooper PA, Dear JP. The effects of air and underwater blast on composite sandwich panels and tubular laminate structures. Exp Mech 2012;52:59-81. doi:10.1007/s11340-011-9506-Z.

[8] Bachynski EE, Motley MR, Young YL. Dynamic hydroelastic scaling of the underwater shock response of composite marine structures. J Appl Mech 2011;79:14501.

[9] Latourte F, Grégoire D, Zenkert D, Wei X, Espinosa HD. Failure mechanisms in composite panels subjected to underwater impulsive loads. J Mech Phys Solids 2011;59:1623-1646. doi:http://dx.doi.org/10.1016/j.jmps.2011.04.013.

[10] Espinosa HD, Lee S, Moldovan N. A novel fluid structure interaction experiment to investigate deformation of structural elements subjected to impulsive loading. Exp Mech 2006;46:805-824. doi:10.1007/s11340-006-0296-7.

[11] Schiffer A, Tagarielli VL, Cocks ACF, Petrini, N. The response of composite plates to underwater blast. In: ASME 2012 international mechanical engineering congress and exposition, volume 8: mechanics of solids, structures and fluids. Houston, TX: ASME, 2012, p. 749. doi:10.1115/IMECE2012-85717.

[12] Avachat S. Experimental and numerical analyses of dynamic deformation and failure in marine structures subjected to underwater impulsive loads. Atlanta, GA: Georgia Institute of Technology, 2012.

[13] Mouritz AP. Ballistic impact and explosive blast resistance of stitched composites. Compos Part B Eng 2001;32:431-439. doi:http://dx.doi.org/10.1016/S1359-8368(01)00015-4. 
[14] Avachat S, Zhou M. Experimental analysis of dynamic deformation and damage in composite sandwich structures subjected to underwater impulsive loads. In: Chalivendra V, Song B, Casem D (eds). Dynamic behavior of materials, volume 1 se-35, conference proceedings of the society for experimental mechanics series. New York: Springer, 2013, pp. 275-286. doi:10.1007/978-1-4614-4238-7_35.

[15] Raimondo L, Iannucci L, Robinson P, Curtis PT. A progressive failure model for meshsize-independent FE analysis of composite laminates subject to low-velocity impact damage. Compos Sc. Techno. 2012;72:624-632. doi:http://dx.doi.org/10.1016/j.compscitech.2012.01.007.

[16] Nguyen VP, Rabczuk T, Bordas S, Duflot M. Meshless methods: a review and computer implementation aspects. Math Comput Sim 2008;79:763-813.

[17] Petschek AG, Libersky L. Cylindrical smoothed particle hydrodynamics. J Comput Phys 1993;109(1):76-83.

[18] Randles PW, Libersky LD. Recent improvements in SPH modeling of hypervelocity impact. Inter J Impact Eng 1997;20:525-532.

[19] Benz W, Asphaug E. Simulations of brittle solids using smooth particle hydrodynamics. Comput Phys Com 1995;87:253-265.

[20] Li S, Hao W, Liu WK. Mesh-free simulations of shear banding in large deformation. Inter J Solids Struct 2000;37:7185-7206.

[21] Li S, Hao W, Liu WK. Numerical simulations of large deformation of thin shell structures using meshfree methods. Comput Mech 2000;25(2-3):102-116. SAME AS [28]

[22] Rabczuk T, Areias PMA, Belytschko T. A simplified meshfree method for shear bands with cohesive surfaces. Inter J Numer Methods Eng 2007;69(5):993-1021. 
[23 Rabczuk T, Samaniego E. Discontinuous modelling of shear bands using adaptive meshfree methods. Comput Methods Appl Mech Eng 2008;197(6-8):641-658.

[24] Rabczuk T, Belytschko T. A three dimensional large deformation meshfree method for arbitrary evolving cracks. Comput Methods Appl Mech Eng 2007;196(29-30): 2777-2799.

[25] Wu Y, Wang DD, Wu CT. Three dimensional fragmentation simulation of concrete structures with a nodally regularized meshfree method. Theor Appl Fract Mech 2014;72:89-99.

[26] Chuzel-Marmot Y, Ortiz R, Combescure A. Three-dimensional SPH-FEM gluing for simulation of fast impacts on concrete slabs. Comput Struct 2011;89:2484-2494.

[27] Rabczuk T, Areias PMA, Belytschko T. A meshfree thin shell method for nonlinear dynamic fracture. Int J Numer Methods Eng 2007;72(5):524-548.

[28] Maurel B, Combescure A. An SPH shell formulation for plasticity and fracture analysis in explicit dynamics. Int J Numer Methods Eng 2008;76:949-971.

[29] Rabczuk T, Gracie R, Song JH, Belytschko T. Immersed particle method for fluid-structure interaction. Int J Numer Methods Eng 2010;81(1):48-71.

[30] Caleyron F, Combescure A, Faucher V, Potapov S. SPH modeling of fluid-solid interaction of dynamic failure analysis of fluid-filled thin shells. J Fluids Struct 2013;39:126-153.

[31] Belytschko T, Guo Y, LiuWK, Xiao SP. A unified stability analysis of meshfree particle methods. Int J Numer Methods Eng 2000:48:1359-1400.

[32] Rabczuk T, Belytschko T, Xiao SP. Stable particle methods based on Lagrangian kernels. Comput Methods Appl Mech Eng 2004;193(12-14):1035-1063.

[33] Sulsky D, Chen Z, Schreyer HL. A particle method for history-dependent materials. Comput Methods Appl Mech Eng 1994;18:179-196. 
[34] Rabczuk T, Belytschko T. Cracking particles: a simplified meshfree method for arbitrary evolving cracks. Int J Numer Methods Eng 2004;61(13):2316-2343.

[35] Rabczuk T, Zi G, Bordas S, Nguyen-Xuan H. A simple and robust three-dimensional cracking-particle method without enrichment. Comput Methods Appl Mech Eng 2010;199(37-40):2437-2455.

[36] Maurel B., Combescure A.: An SPH shell formulation for plasticity and fracture analysis in explicit dynamics, Int J Numer Methods Eng 2008;76:949971.

[37] Silling SA, Reformulation of elasticity theory for discontinuities and long-range forces. J Mech Phys Solids 2000;48:175-209.

[38] Silling SA, Epton M, Weckner O, Xu J, Askari A. Peridynamics states and constitutive modeling. J Elast 2007;88:151-184.

[39] Oterkus E, Madenci E. Peridynamic analysis of fiber reinforced composite materials. J Mech Mater Struct 2012;7:45-84.

[40] Oterkus E, Madenci E, Weckner O, Silling S, Bogert P, Tessler A. Combined finite element and peridynamic analyses for predicting failure in a stiffened composite curved panel with a central slot. Compos Struct 2012;94:839-850.

[41] Agwai A, Guven I, Madenci E. predicting crack propagation with peridynamics: a comparative study. Int J Fract 2011;171:65-78.

[42] Kilic B, Madenci E., Coupling of peridynamic theory and finite element method. J Mech Mater Struct 2010;5:707-733.

[43] Kilic B, Madenci E. Structural stability and failure analysis using peridynamic theory. Int J Non-Linear Mech 2009:44:845-854. 
[44] Kilic B, Agwai A, Madenci E. Peridynamic theory for progressive damage prediction in centre-cracked composite laminates. Compos Struct 2009;90:141-151.

[45] Hu YL, De Carvalho NV, Madenci, E. Peridynamic modeling of delamination growth in composite laminates. Compos Struct 2015;32:610-620.

[46] Madenci E, Oterkus E. Peridynamic theory and its applications, peridynamic theory and its applications. New York: Springer, 2014. doi:10.1007/978-1-4614-8465-3.

[47] Colavito K. Peridynamics for failure and residual strength prediction of fiber-reinforced composites. Tucson, AZ: The University of Arizona, 2013.

[48] Silling SA, Askari E. A meshfree method based on the peridynamic model of solid mechanics. Comput Struct 2005;83:1526-1535.

[49] Colavito KW, Kilic B, Celik E, Madenci E, Askari E, Silling, S. Effect of void content on stiffness and strength of composites by a peridynamic analysis and static indentation test. Honolulu, HI: 48th AIAA/ASME/ASCE/AHS/ASC Structures, Structural Dynamics, and Materials Conference, 2007, Paper No. AIAA 2007-2251.

[50] Colavito KW, Kilic B, Celik E, Madenci E, Askari, Silling S., Effect of nano particles on stiffness and impact strength of composites. Honolulu, HI: $48^{\text {th }}$ AIAA/ASME/ASCE/AHS/ASC Structures, Structural Dynamics, and Materials Conference, 2007, Paper No. AIAA 2007-2021.

[51] Hu W, Ha YD, Bobaru F. Peridynamic model for dynamic fracture in unidirectional fiberreinforced composites. Comput Methods Appl Mech Eng 2012;217-220:247-261.

[52] Comtois JLR, Edwards MR, Oakes MC. The effect of explosives on polymer matrix composite laminates. Compos Part A Appl Sci Manuf 1999;30:181-190. doi:http://dx.doi.org/10.1016/S1359-835X(98)00172-9. 